\title{
Design and Optimization of the Lighting Environment for the Brand Clothing Stores Based on DIALUX Software
}

\author{
Xiao-cong $\mathrm{Hu}$ \\ School of Xiamen University of Technology, Xiamen 361024, China. \\ Xiaocong56@sohu.com
}

\begin{abstract}
Lighting design is an important means of creating a space atmosphere of clothing stores, good lighting effects can effectively enhance the brand image value. In order to achieve the balance of economy and art of space lighting, clothing store lighting environment often need to consider different design goals, it is necessary to be able to attract customers to attract attention, promote consumption, but also to achieve moderate economic effects of lighting, Must involve complex lighting calculations and simulation work. DIALUX lighting software can accurately simulate the indoor and outdoor space lighting design, the article Adidas clothing store lighting as the object of study, based on the brand image and product display understanding, detailed analysis of space lighting design problems, the use of DIALUX lighting software Simulate and calculate a reasonable space lighting program, and according to the calculation results reasonable configuration of various parameters of lighting, in order to achieve the best program of function and brand display.
\end{abstract}

Key words.kindergarten classroom;lighting design; DIALUX;design optimization;

\section{INTRODUCTION}

The lighting design in the store determines the customer to make the appropriate purchase behavior, this visual impression is often determined by the lighting design configuration. In the lighting design needs through the clever lighting configuration, to create a characteristic and attractive, pleasant and relaxed and safe business space environment. [1] clothing store is an important place for product marketing, on the one hand, lighting design not only to meet the basic functional requirements, but also highlight the brand culture and style positioning, to attract and guide consumers shopping intention; the other hand, Space lighting design According to the clothing store within the regional clothing wall, POP, image wall, showcase, exhibitive platform functional differences, will be divided into different lighting levels, according to different design goals need to configure a variety of lamps. Therefore, the lighting design has shifted from a single lighting function to comfort, art and energy efficient, in the design of the program by virtue of experience is difficult to obtain the ideal illumination distribution and other quantitative indicators, which will use such as DIALUX lighting software Simulation, so as to refer to the standard specification to obtain the best balance between the design effect and the economic cost.

\section{Clothing store lighting design of the relevant norms and requirements}

In order to improve the quality of lighting, each country has developed a lighting standard that conforms to its own national conditions. It not only guides and regulates the design and implementation of lighting engineering, but also reflects the lighting level of a country or region. The study of clothing store lighting will refer to the International Lighting Engineering Committee of the CIE S 008 / E-2001 "indoor workplace lighting", the People's Republic of China national standard GB/T 26189-2010 "indoor workplace lighting" and GB50034-2013 "Architectural lighting design standards" three standard specifications. The specification clearly states that good workplace lighting is not just about providing a good job visibility, it is vital to work comfortably and comfortably, and lighting must meet the needs of visual comfort, visual efficacy and visual safety, with a major reference to the 
brightness distribution, [2] In addition, from the green energy saving and visual comfort point of view, illuminance, illumination uniformity (Uo) and the lighting power density (LPD) three items are the focus of the store lighting need to refer to the indicators.TABLE1

TABLE 1. Store building lighting standard value.

\begin{tabular}{|c|c|c|c|c|c|}
\hline Room or Place & $\begin{array}{l}\text { Reference plane } \\
\text { and height }\end{array}$ & $\begin{array}{c}\text { Standard } \\
\text { value of } \\
\text { illuminatio } \\
\mathbf{n}(\mathrm{LX})\end{array}$ & $\begin{array}{l}\text { Illumination } \\
\text { density limit } \\
\quad(\mathrm{W} / \mathrm{m} 2)\end{array}$ & $\begin{array}{c}\text { Illumination } \\
\text { uniformity } \\
\text { (UO) }\end{array}$ & $\mathbf{R a}$ \\
\hline $\begin{array}{c}\text { General store business } \\
\text { hall }\end{array}$ & $\begin{array}{c}0.75 \mathrm{~m} \text { horizontal } \\
\text { plane }\end{array}$ & 300 & $\leqq 9.0$ & 0.60 & 80 \\
\hline Senior store business hall & $\begin{array}{l}0.75 \mathrm{~m} \text { horizontal } \\
\text { plane }\end{array}$ & 500 & $\leqq 14.5$ & 0.60 & 80 \\
\hline Store business hall & $\begin{array}{l}0.75 \mathrm{~m} \text { horizontal } \\
\text { plane }\end{array}$ & 500 & $\leqq 10.0$ & 0.60 & 80 \\
\hline Cashier & $\begin{array}{c}\text { Counter } \\
\text { horizontal plane }\end{array}$ & 500 & - & 0.60 & 80 \\
\hline
\end{tabular}

Adidas clothing store belongs to the store business hall, therefore, the illumination will refer to the store business hall illumination value of 500LX, illumination uniformity in the CIE standard S 008 / E-2001 "indoor workplace lighting" in the provisions of 0.70 , And China's standard set to 0.6 , lighting power density should be $\leqq 10.0$, color is 80 , the evaluation of research results will be based on these indicators as a reference.

Modern clothing store diversified business model and design style, requiring space lighting presents a rich level, often composed of a variety of types of lighting, the formation of different lighting effects, can be divided into: window lighting, entrance lighting, store general Lighting, lighting and special lighting (such as cash registers, etc.), [3] of which, the entrance lighting should generally be higher than the average indoor illumination, about 1.5-2 times the light should be more gathered some; general lighting is to light The uniform lighting set in the whole place, belonging to non-directional lighting, requires a better illumination uniformity, proper color temperature and higher light color; focus on lighting is to highlight the goods in the environment, the ratio of the general lighting is 5: 1 to 15: 1, this contrast can shape the high value of goods, attract customers attention to the goods, causing the desire to buy; cashier is more need to form a visual focus, illumination and lighting should be different from the store in general illumination.

\section{DIALUX software and operation flow procedure}

DIALUX is a German developed with 3D simulation lighting design free lighting software to support the light source data (IES, CIE, CIBSE, ULD, PDF, etc.), and gradually with Philips, BEGA, THORN, ERCO, OSRAM, BJB , Meyer, Louis Poulsen and other world-renowned lighting manufacturers, and these mainstream lighting manufacturers database friendly and compatible, you can easily achieve the lighting design of the precise simulation and calculation, is now more and more widely used. DIALUX4.12 version after the introduction of the DIALUX evo version, the software interface and mode of operation have been simplified, adding more automated operations, and provide three-dimensional simulation lamp model, DWG import and export, 3D renderings, pseudo- Highresolution trace tracking, power consumption and accurate illumination values to see if it is to the lighting standard so that the lighting design becomes more intuitive and intelligent.

Fast and accurate light environment simulation requires accurate model and the correct input of the actual parameters, the study selected DIALUX evo software has similar to the basic functions of three-dimensional software, first, the introduction of simplified CAD drawings, according to the size of the drawings using the auxiliary line to establish Building the model, set the height of the floor, and then cut into the room space, add the necessary doors and windows, furniture and other components; second, for the space specified material, and the use of lighting manufacturers to provide plug-in placement lamp, the initial light source illumination; , Calculate the parameters of space lighting, generate and export the calculation report.

\section{Adidas clothing store lighting design and optimization}

\section{A. Adidas clothing store lighting design analysis}

Case study Adidas clothing store total area of $177.7 \mathrm{~m}^{2}$, of which the business area of $150.8 \mathrm{~m}^{2}$, warehouse area of $23.5 \mathrm{~m}^{2}$, dressing area of $3.4 \mathrm{~m}^{2}$, business area based on the different forms of goods display, divided into Nakajima display area, Display area, the wall display area and POP display wall, the wall above $2500 \mathrm{~mm}$ and the top of the black nitro paint, the following is the use of gray nitro paint, ground island area with dark gray tiles, wall 
area with light gray tiles, The overall design of the brand distinctive features, gray and black main colors for the diverse colors of clothing products provide a good basis.

Lighting design first detailed analysis of the store space flow line and space layout, the case is located within the mall, $80 \%$ of the flow is moving from the left to the right, the store space using the layout of the island, the use of exhibition, exhibition wall.Therefore, the lighting should pay attention to highlight the overall display area in the island, and the focus of the booth, the model booth, the exhibition booth, the booth, Second, according to the color of the space itself to determine the color of the overall lighting, because Adidas sports clothing color is more abundant, therefore, should be the overall light color temperature positioning 3200 - the color of the overall color, $4000 \mathrm{~K}$ or so, in order to highlight the color temperature through the warm white color clothing itself; Finally, the choice of light type, in addition to the middle of the three lights with T5, 15W fluorescent lamp, wall display area set up 35W track spotlights, the other recommended the use of high efficiency Light source, and color rendering are more than 80, such as low-power high-color gas discharge light source, including $2 \times 20 \mathrm{~W}$ double metal halide lamp, $2 \times 20 \mathrm{~W}$ embedded metal halide lamp, both to achieve better color Sex, but also to lower maintenance costs to achieve the purpose of green design.

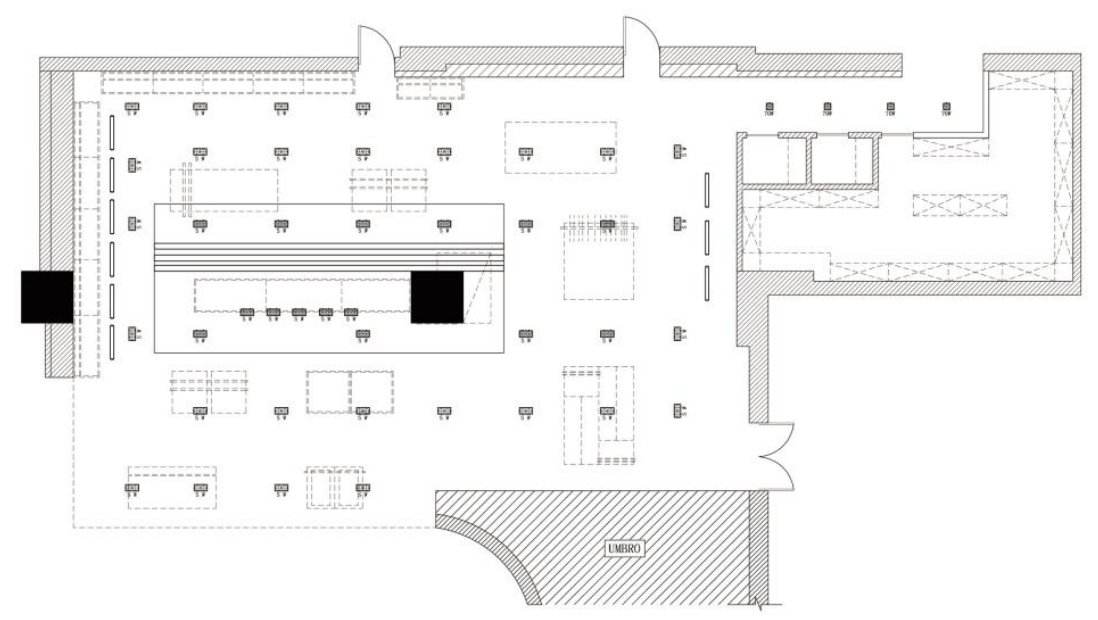

FIGURE1. Lighting design layout

\section{B. Lighting Test and Optimization Based on DIALUX evo Software}

The test of the use of lamps for the OPPLE and NVC two brands, including NVC70061622 NLED551N $1 \times$ 20W $24^{\circ} 4000 \mathrm{~K}$ high-power single-head grille spotlights, Opple140001937 MTH1131-WH-35W-30, Opple140002643 MDP11318V-YD, Opple40042051 MZ1200-D15Z - Ming public -840 and other four lamps; material ground using light gray tiles, walls with gray paint, the top and column with black paint. Basically in line with Adidas store unified brand image,

Through the software test report can be seen, the store sales space surface of the average illumination value of $504 \mathrm{LX}$, lighting density limit $2.65 \mathrm{~W} / \mathrm{m} 2$, illumination uniformity of 0.32 , color is 83 , with reference to regulatory requirements, Lighting density limits and color rendering have reached the store lighting requirements, but the illumination uniformity of only 0.32 , significantly lower than the specification of the requirements of 0.6 , and from the input of the isothere map can also be seen, part of the regional illumination to 750LX, but the collection area of the regional illumination value of only 100LX, which clearly shows the spatial illumination distribution is uneven, in actual use is very easy to produce shadow area and visual fatigue, but also bring unnecessary power loss. 


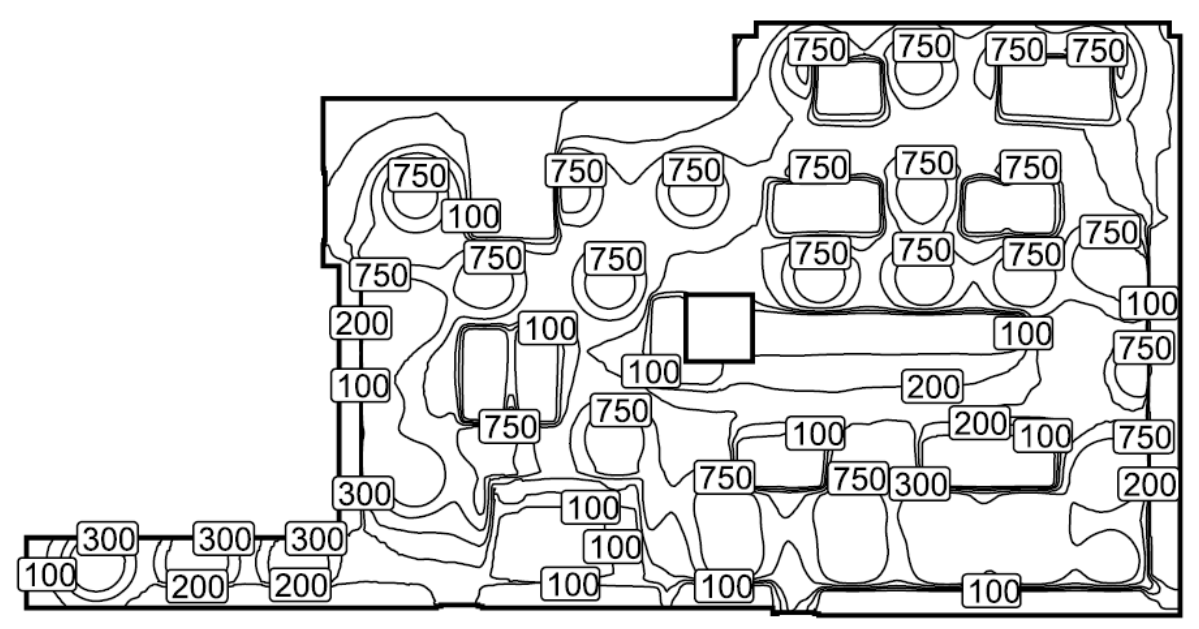

FIGURE 2. Initial test and other illumination chart

Therefore, for the software test reflects the illumination distribution problem, need to be adjusted from the following two aspects, first, according to the spatial positioning of the region to adjust the number of lamps, such as the cash register area should increase the lamp, because this area not only in Space has a clear functional requirements, but also the image of the store an important area, therefore, the illumination should be appropriate to improve, but to take into account the surrounding display cabinet above the spotlights lighting; and by the wall of the exhibition and the entrance should be appropriate to reduce the lamp The number of lighting at the entrance can not exceed the brightness of the window, otherwise it can not achieve the window display effect, the wall display of the illumination should be appropriately reduced, in line with regulatory requirements can be achieved to achieve the purpose of energy saving; Second, adjust the lamp position, The island area of the main display clothing booths and models, lighting should allow consumers to fully feel the space to pass the brand concept and Adidas corporate culture, strengthen the first impression and intuition to attract, if the illumination distribution is uneven, and contrast is large Can not reflect the value of goods, which need to adjust the lamp position, so that the illumination distribution to achieve a balanced.

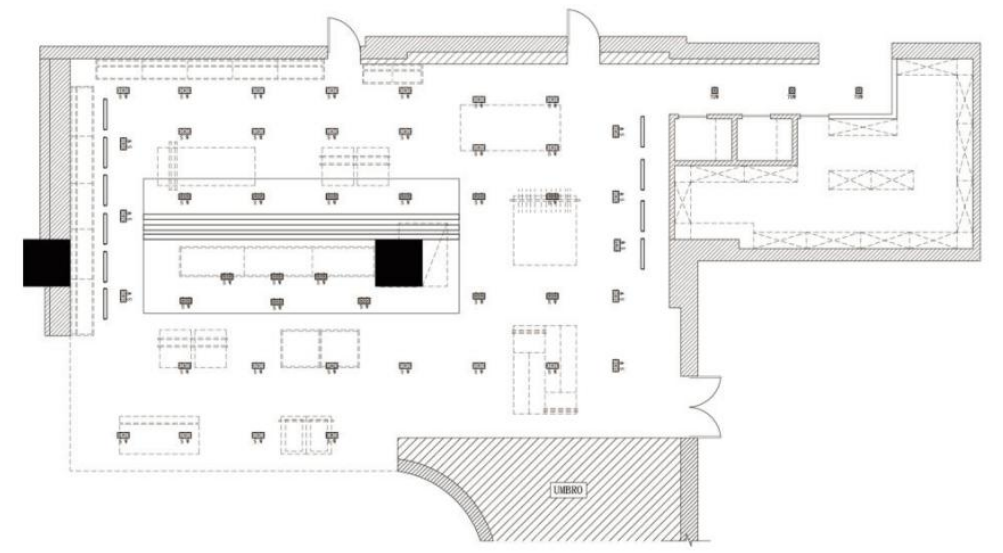

FIGURE 3. Optimized lighting design layout

After the optimization of the use of DIALUX evo test results show: the collection area of the illumination from the previous 100LX increased to 500LX, wall exhibition area of the illumination from the previous 750LX reduced to 500LX, the island area of illumination from 200LX increased to $450 \mathrm{LX}$, Basically in line with the expected objectives. However, the regional illumination increased at the same time, the average illumination from the previous 504LX increased to 586LX, slightly higher than the requirements of the specification, will increase the number of energy consumption, but taking into account the test scene is not placed clothing products, material and the actual design is not complete Consistent, therefore, can be basically determined that the test results are valid. 


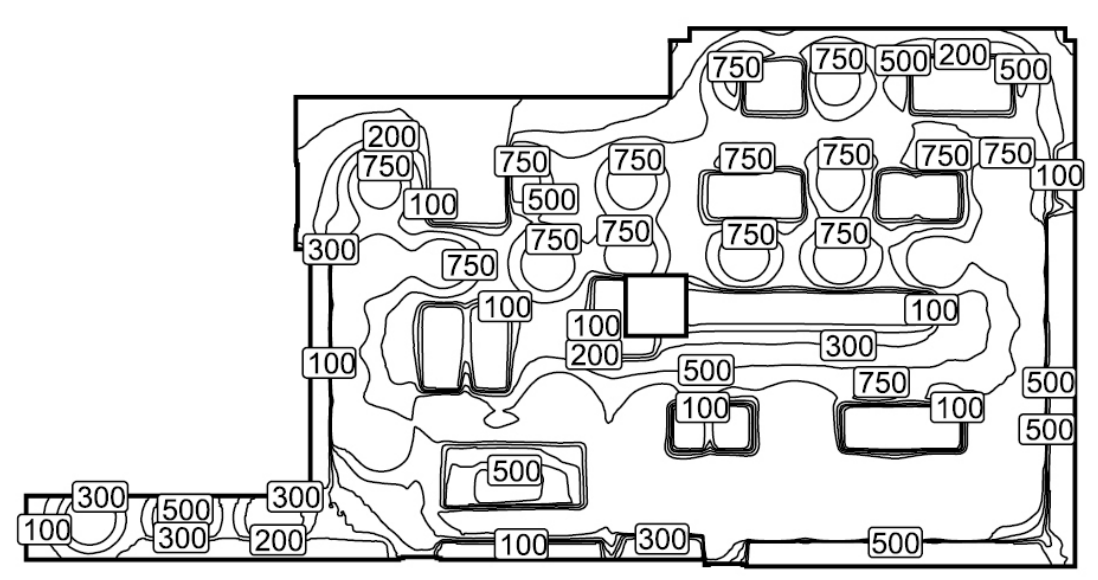

FIGURE 4. Optimized illumination chart

\section{CONCLUSION}

The powerful functionality of the auxiliary design software is, on the one hand, reflected in the flexibility and effectiveness of the problem of dealing with the changing requirements of the environment, and on the other hand, the rationality and logic of its work. The DIALUX evo lighting design software is used to evaluate the lighting effect of Adidas store, and the parameters of the illumination evaluation system can be calculated quickly and accurately, which can make up the deficiency caused by the experience design. Therefore, from the whole process of the case test, DIALUX evo software from the initial two plane model to the model of construction, and then to the lighting, furniture selection and arrangement, and finally for the lighting design to provide close to the real light environment, and provide Personalized parameters of the report, each process are completed in the specified area, and some special settings will be hidden in the toolbar or special mode, the overall interface layout is very userfriendly, master the use of this software will be conducive to Improve the quality of lighting design, and international advanced design level.

\section{ACKNOWLEDGMENTS}

This work was financially supported by 2016 Education and Teaching Reform Project of Xiamen University of Technology fund (JGY201678).

\section{REFERENCES}

1. JIN Xu-dong.Analysis and Research on the Store Lighting Design,J.PACKAGING ENGINEERING,2011(09):131-134.

2. YUAN Qiao.International Lighting Commission Lighting Standards-Indoor Workplace Lighting,J.CHINA ILLUMINATING ENGINEERING JOURNAL,2002(12):55-60.

3. BEIJING Lighting Society Lighting Design Specialized Committee.Lighting Design Manual,M.BEIJING: China Electric Power Press,2006. 